\title{
The Kamenev type interval oscillation criteria of mixed nonlinear impulsive differential equations under variable delay effects
}

\section{Xiaoliang Zhou ${ }^{1 *}$ (D), Changdong $\mathrm{Liu}^{2}$ and Ruyun Chen ${ }^{2}$}

"Correspondence:

zxImath@163.com

${ }^{1}$ Department of Mathematics,

Lingnan Normal University,

Zhanjiang, P.R. China

Full list of author information is

available at the end of the article

\section{Springer}

\begin{abstract}
In this paper, a class of mixed nonlinear impulsive differential equations is studied. When the delay $\sigma(t)$ is variable, each given interval is divided into two parts on which the quotients of $x(t-\sigma(t))$ and $x(t)$ are estimated. Then, by introducing binary auxiliary functions and using the Riccati transformation, several Kamenev type interval oscillation criteria are established. The well-known results obtained by Liu and Xu (Appl. Math. Comput. 215:283-291, 2009) for $\sigma(t)=0$ and by Guo et al. (Abstr. Appl. Anal. 2012:351709, 2012) for $\sigma(t)=\sigma_{0}\left(\sigma_{0} \geq 0\right)$ are developed. Moreover, an example illustrating the effectiveness and non-emptiness of our results is also given.
\end{abstract}

Keywords: Interval oscillation; Impulsive differential equation; Variable delay; Interval delay function

\section{Introduction}

We consider the following mixed nonlinear impulsive differential equations with variable delay:

$$
\begin{aligned}
& \left(r(t) \Phi_{\alpha}\left(x^{\prime}(t)\right)\right)^{\prime}+p_{0}(t) \Phi_{\alpha}(x(t))+\sum_{i=1}^{n} p_{i}(t) \Phi_{\beta_{i}}(x(t-\sigma(t)))=f(t), \quad t \geq t_{0}, t \neq \tau_{k}, \\
& x\left(\tau_{k}^{+}\right)=a_{k} x\left(\tau_{k}\right), \quad x^{\prime}\left(\tau_{k}^{+}\right)=b_{k} x^{\prime}\left(\tau_{k}\right), \quad k=1,2, \ldots,
\end{aligned}
$$

where $\Phi_{*}(s)=|s|^{*-1} s,\left\{\tau_{k}\right\}$ denotes the impulse moments, $0 \leq t_{0}<\tau_{1}<\tau_{2}<\cdots<\tau_{k}<\cdots$ and $\lim _{k \rightarrow \infty} \tau_{k}=\infty,\left\{a_{k}\right\}$ and $\left\{b_{k}\right\}$ are real constant sequences and $b_{k} \geq a_{k}>0$ for $k=$ $1,2, \ldots, \sigma(t) \in C\left(\left[t_{0}, \infty\right)\right)$ and there exists a nonnegative constant $\sigma_{0}$ such that $0 \leq \sigma(t) \leq$ $\sigma_{0}$ for all $t \geq t_{0}, r(t) \in C^{1}\left(\left[t_{0}, \infty\right),(0, \infty)\right)$ is nondecreasing.

For some particular cases of (1), many authors have devoted work to the interval oscillation problem (see [3-13]). Particularly, when $\alpha=1, a_{k}=b_{k}=1$ and $\sigma(t)=0$, (1) reduces to the mixed type Emden-Fowler equation

$$
\left(r(t) x^{\prime}(t)\right)^{\prime}+p_{0}(t) x(t)+\sum_{i=1}^{n} p_{i}(t) \Phi_{\beta_{i}}(x(t))=f(t)
$$

(0) The Author(s) 2019. This article is distributed under the terms of the Creative Commons Attribution 4.0 International License (http://creativecommons.org/licenses/by/4.0/), which permits unrestricted use, distribution, and reproduction in any medium, provided you give appropriate credit to the original author(s) and the source, provide a link to the Creative Commons license, and indicate if changes were made. 
which was given much attention due to the effect of modeling the growth of bacteria population with competitive species. For example, in [14] and [15], the authors established interval oscillation theorems for (2) which improved the well-known criteria of [16] and [17]. For additional studies of Emden-Fowler differential equations, see [18-20].

In [1], the authors considered (2) with impulse effects,

$$
\begin{aligned}
& \left(r(t) x^{\prime}(t)\right)^{\prime}+p_{0}(t) x(t)+\sum_{i=1}^{n} p_{i}(t) \Phi_{\beta_{i}}(x(t))=f(t), \quad t \geq t_{0}, t \neq \tau_{k}, \\
& x\left(\tau_{k}^{+}\right)=a_{k} x\left(\tau_{k}\right), \quad x^{\prime}\left(\tau_{k}^{+}\right)=b_{k} x^{\prime}\left(\tau_{k}\right), \quad k=1,2, \ldots,
\end{aligned}
$$

and established some interval oscillation results which extended those of $[14,15]$ and $[21]$. When $\sigma(t)=0$, (1) becomes the following impulse equations without delay:

$$
\begin{aligned}
& \left(r(t) \Phi_{\alpha}\left(x^{\prime}(t)\right)\right)^{\prime}+p_{0}(t) \Phi_{\alpha}(x(t))+\sum_{i=1}^{n} p_{i}(t) \Phi_{\beta_{i}}(x(t))=f(t), \quad t \geq t_{0}, t \neq \tau_{k}, \\
& x\left(\tau_{k}^{+}\right)=a_{k} x\left(\tau_{k}\right), \quad x^{\prime}\left(\tau_{k}^{+}\right)=b_{k} x^{\prime}\left(\tau_{k}\right), \quad k=1,2, \ldots
\end{aligned}
$$

In [22], Özbekler and Zafer investigated (4). They considered the coefficients $p_{i}(t)(i=$ $1,2, \ldots, n)$ satisfying two cases: (i) $p_{i}(t) \geq 0$ for $i=1,2, \ldots, n$ and (ii) $p_{i}(t) \geq 0$ for $i=$ $1,2, \ldots, m ; p_{i}(t)$ are allowed to be negative for $i=m+1, \ldots, n$ and obtained several interval oscillation results which recovered the early ones in [8] and [14].

When $\sigma(t)$ is a nonnegative constant, i.e., $\sigma(t)=\sigma_{0}\left(\sigma_{0} \geq 0\right)$, by idea of [23], Guo et al. [2] studied (1) and developed the results of [1, 22, 24].

Recently, in [25], the authors studied (1) with the assumption of delay $\sigma(t)$ being variable. They used Riccati transformation and univariate $\omega$ functions to obtain some generalized interval oscillation results.

In this paper, we continue the discussion of the interval oscillation of (1). Unlike the methods of $[22,25]$, we introduce a binary auxiliary function, divide each given interval into two parts and then estimate the quotients of $x(t-\sigma(t))$ and $x(t)$. Due to the considered delay being variable, the results obtained here are the development of some well-known ones, such as in [1] and [2]. Moreover, we also give an example to illustrate the effectiveness and non-emptiness of our results.

\section{Main results}

First, we define a functional space $C_{-}(I, \mathbb{R})$ as follows:

$$
\begin{aligned}
C_{-}(I, \mathbb{R}):= & \left\{y: I \rightarrow \mathbb{R} \mid I \text { is a real interval, } y \text { is continuous on } I \backslash\left\{t_{i}\right\}\right. \text { and } \\
& \left.y\left(t_{i}^{-}\right)=y\left(t_{i}\right), i \in \mathbb{N}\right\} .
\end{aligned}
$$

In the following, we always assume:

(A) the exponents satisfy $\beta_{1}>\cdots>\beta_{m}>\alpha>\beta_{m+1}>\cdots>\beta_{n}>0$;

$$
f(t), p_{i}(t) \in C_{-}\left(\left[t_{0}, \infty\right), \mathbb{R}\right), i=0,1, \ldots, n ; \tau_{k+1}-\tau_{k}>\sigma_{0} \text { for all } k=1,2, \ldots
$$

Let $k(s)=\max \left\{i: t_{0}<\tau_{i}<s\right\}$. For any given intervals $\left[c_{j}, d_{j}\right](j=1,2)$, we suppose that $k\left(c_{j}\right)<k\left(d_{j}\right)(j=1,2)$, then there exist impulse moments $\tau_{k\left(c_{j}\right)+1}, \ldots, \tau_{k\left(d_{j}\right)}$ in $\left[c_{j}, d_{j}\right](j=1,2)$ and we have the following cases to consider. 
$\left(S_{1}\right) \tau_{k\left(c_{j}\right)}+\sigma_{0}<c_{j}$ and $\tau_{k\left(d_{j}\right)}+\sigma_{0}<d_{j} ;$

$\left(S_{2}\right) \tau_{k\left(c_{j}\right)}+\sigma_{0}<c_{j}$ and $\tau_{k\left(d_{j}\right)}+\sigma_{0}>d_{j} ;$

$\left(S_{3}\right) \tau_{k\left(c_{j}\right)}+\sigma_{0}>c_{j}$ and $\tau_{k\left(d_{j}\right)}+\sigma_{0}>d_{j}$

$\left(S_{4}\right) \tau_{k\left(c_{j}\right)}+\sigma_{0}>c_{j}$ and $\tau_{k\left(d_{j}\right)}+\sigma_{0}<d_{j}$.

We further assume that there exist points $\delta_{j} \in\left(c_{j}, d_{j}\right) \backslash\left\{\tau_{k}\right\}(j=1,2)$ which divide intervals $\left[c_{j}, d_{j}\right]$ into two parts $\left[c_{j}, \delta_{j}\right]$ and $\left[\delta_{j}, d_{j}\right]$. In view of whether or not there are impulsive moments of $x(t)$ in $\left[c_{j}, \delta_{j}\right]$ and $\left[\delta_{j}, d_{j}\right]$, we should consider the following cases.

$\left(\bar{S}_{1}\right) \quad k\left(c_{j}\right)<k\left(\delta_{j}\right)<k\left(d_{j}\right)$;

$\left(\bar{S}_{2}\right) k\left(c_{j}\right)=k\left(\delta_{j}\right)<k\left(d_{j}\right)$;

$\left(\bar{S}_{3}\right) k\left(c_{j}\right)<k\left(\delta_{j}\right)=k\left(d_{j}\right)$.

We define a interval delay function ([12]):

$$
D_{k}(t)=t-\tau_{k}-\sigma(t), \quad t \in\left(\tau_{k}, \tau_{k+1}\right], k=1,2, \ldots
$$

and we assume there is a point $t_{k} \in\left(\tau_{k}, \tau_{k+1}\right]$ such that $D_{k}\left(t_{k}\right)=0, D_{k}(t)<0$ for $t \in\left(\tau_{k}, t_{k}\right)$ and $D_{k}(t)>0$ for $t \in\left(t_{k}, \tau_{k+1}\right]$.

Moreover, for the relationship of the division point $\delta_{j}$ and the zero point $t_{k\left(\delta_{j}\right)}$ of $D_{k\left(\delta_{j}\right)}$ on $\left[\tau_{k\left(\delta_{j}\right)}, \tau_{k\left(\delta_{j}\right)+1}\right]$ we should have

$\left(\overline{\bar{S}}_{1}\right) t_{k\left(\delta_{j}\right)}<\delta_{j}$;

$\left(\overline{\bar{S}}_{2}\right) t_{k\left(\delta_{j}\right)}>\delta_{j}$; or

$\left(\overline{\bar{S}}_{3}\right) t_{k\left(\delta_{j}\right)}=\delta_{j}$.

We only consider the case of combination of $\left(S_{1}\right)$ with $\left(\bar{S}_{1}\right)$ and $\left(\overline{\bar{S}}_{1}\right)$. For the other cases, the discussion will be omitted here.

Lemma 2.1 Assume that, for any $T \geq t_{0}$, there exist $T<c_{1}-\sigma_{0}<c_{1}<\delta_{1}<d_{1}$ and

$$
f(t) \leq 0, \quad p_{i}(t) \geq 0, \quad t \in\left[c_{1}-\sigma_{0}, d_{1}\right] \backslash\left\{\tau_{k}\right\}, i=0,1,2, \ldots, n,
$$

and $t_{k}$ is a zero point of $D_{k}\left(t_{k}\right)$ in $\left(\tau_{k}, \tau_{k+1}\right]$. If $x(t)$ is a positive solution of $(1)$, then the ratio $x(t-\sigma(t)) / x(t)$ will be estimated as follows:
(a) $\frac{x(t-\sigma(t))}{x(t)}>\frac{t-\tau_{k}-\sigma(t)}{t-\tau_{k}}, t \in\left(t_{k}, \tau_{k+1}\right]$ for $k=k\left(c_{1}\right)+1, \ldots, k\left(d_{1}\right)-1, k \neq k\left(\delta_{1}\right)$;
(b) $\frac{x(t-\sigma(t))}{x(t)}>\frac{t-\tau_{k}}{b_{k}\left(t+\sigma(t)-\tau_{k}\right)}, t \in\left(\tau_{k}, t_{k}\right]$ for $k=k\left(c_{1}\right)+1, \ldots, k\left(d_{1}\right)$;
(c) $\frac{x(t-\sigma(t))}{x(t)}>\frac{t-\tau_{k\left(\delta_{1}\right)}-\sigma(t)}{t-\tau_{k\left(\delta_{1}\right)}}, t \in\left(t_{k\left(\delta_{1}\right)}, \delta_{1}\right]$;
(d) $\frac{x(t-\sigma(t))}{x(t)}>\frac{t-\tau_{k\left(d_{1}\right)}-\sigma(t)}{t-\tau_{k\left(d_{1}\right)}}, t \in\left(t_{k\left(d_{1}\right)}, d_{1}\right]$;
(e) $\frac{x(t-\sigma(t))}{x(t)}>\frac{t-\tau_{k\left(\delta_{1}\right)}-\sigma(t)}{t-\tau_{k\left(\delta_{1}\right)}}, t \in\left(\delta_{1}, \tau_{k\left(\delta_{1}\right)+1}\right]$;
(f) $\frac{x(t-\sigma(t))}{x(t)}>\frac{t-\tau_{k\left(c_{1}\right)}-\sigma(t)}{t-\tau_{k\left(c_{1}\right)}}, t \in\left[c_{1}, \tau_{k\left(c_{1}\right)+1}\right]$.

Proof From (1), (5) and (A), we obtain, for $t \in\left[c_{1}, d_{1}\right] \backslash\left\{\tau_{k}\right\}$,

$$
\left(r(t) \Phi_{\alpha}\left(x^{\prime}(t)\right)\right)^{\prime}=f(t)-p_{0}(t) \Phi_{\alpha}(x(t))-\sum_{i=1}^{n} p_{i}(t) \Phi_{\beta_{i}}(x(t-\sigma(t))) \leq 0 .
$$

Hence $r(t) \Phi_{\alpha}\left(x^{\prime}(t)\right)$ is nonincreasing on the interval $\left[c_{1}, d_{1}\right] \backslash\left\{\tau_{k}\right\}$. Next, we give the proof of case (a) only. For the other cases, the proof is similar and will be omitted. 
If $t_{k}<t \leq \tau_{k+1}$, from $D_{k}(t)>0$, we know $(t-\sigma(t), t) \subset\left(\tau_{k}, \tau_{k+1}\right]$. Thus there is no impulse moment in $(t-\sigma(t), t)$. Therefore, for any $s \in(t-\sigma(t), t)$, there exists a $\xi_{k} \in\left(\tau_{k}, s\right)$ such that $x(s)-x\left(\tau_{k}^{+}\right)=x^{\prime}\left(\xi_{k}\right)\left(s-\tau_{k}\right)$. Since $x\left(\tau_{k}^{+}\right)>0, r(s)$ is nondecreasing, $\Phi_{\alpha}(\cdot)$ is an increasing function and $r(t) \Phi_{\alpha}\left(x^{\prime}(t)\right)$ is nonincreasing on $\left(\tau_{k}, \tau_{k+1}\right]$, we have

$$
\begin{aligned}
\Phi_{\alpha}(x(s)) & \geq \frac{r\left(\xi_{k}\right)}{r(s)} \Phi_{\alpha}(x(s))>\frac{r\left(\xi_{k}\right)}{r(s)} \Phi_{\alpha}\left(x^{\prime}\left(\xi_{k}\right)\left(s-\tau_{k}\right)\right)=\frac{r\left(\xi_{k}\right) \Phi_{\alpha}\left(x^{\prime}\left(\xi_{k}\right)\right)}{r(s)}\left(s-\tau_{k}\right)^{\alpha} \\
& \geq \frac{r(s) \Phi_{\alpha}\left(x^{\prime}(s)\right)}{r(s)}\left(s-\tau_{k}\right)^{\alpha}=\Phi_{\alpha}\left(x^{\prime}(s)\left(s-\tau_{k}\right)\right) .
\end{aligned}
$$

Therefore,

$$
\frac{x^{\prime}(s)}{x(s)}<\frac{1}{s-\tau_{k}}
$$

Integrating both sides of the above inequality from $t-\sigma(t)$ to $t$, we obtain

$$
\frac{x(t-\sigma(t))}{x(t)}>\frac{t-\tau_{k}-\sigma(t)}{t-\tau_{k}}, \quad t \in\left(t_{k}, \tau_{k+1}\right] .
$$

The proof is completed.

Lemma 2.2 Assume that, for any $T \geq t_{0}$, there exist $T<c_{2}-\sigma_{0}<c_{2}<\delta_{2}<d_{2}$ and

$$
f(t) \geq 0, \quad p_{i}(t) \geq 0, \quad t \in\left[c_{2}-\sigma_{0}, d_{2}\right] \backslash\left\{\tau_{k}\right\}, \quad i=0,1,2, \ldots, n,
$$

and $t_{k}$ is a zero point of $D_{k}\left(t_{k}\right)$ in $\left(\tau_{k}, \tau_{k+1}\right]$. If $x(t)$ is a negative solution of $(1)$, then estimations (a)-(f) in Lemma 2.1 are correct with the replacement of $c_{1}, d_{1}$ and $\delta_{1}$ by $c_{2}, d_{2}$ and $\delta_{2}$, respectively.

The proof of Lemma 2.2 is similar to that of Lemma 2.1 and will be omitted.

Lemma 2.3 Assume that for any $T \geq t_{0}$ there exists $T<c_{1}-\sigma_{0}<c_{1}<d_{1}$ and (5) holds. If $x(t)$ is a positive solution of $(1)$ and $u(t)$ is defined by

$$
u(t):=\frac{r(t) \Phi_{\alpha}\left(x^{\prime}(t)\right)}{\Phi_{\alpha}(x(t))}, \quad t \in\left[c_{1}, d_{1}\right]
$$

then we have the following estimations of $u(t)$ :

(g) $u\left(\tau_{k+1}\right) \leq \frac{\widetilde{r}}{\left(\tau_{k+1}-\tau_{k}\right)^{\alpha}}, \tau_{k+1} \in\left[c_{1}, d_{1}\right], k=k\left(c_{1}\right)+1, \ldots, k\left(d_{1}\right)-1, k \neq k\left(\delta_{1}\right)$;

(h) $u\left(\tau_{k\left(c_{1}\right)+1}\right) \leq \frac{\widetilde{r}}{\left(\tau_{k\left(c_{1}\right)+1}-c_{1}\right)^{\alpha}}, \tau_{k\left(c_{1}\right)+1} \in\left[c_{1}, d_{1}\right]$;

(i) $u\left(\tau_{k\left(\delta_{1}\right)+1}\right) \leq \frac{\widetilde{r}}{\left(\tau_{k\left(\delta_{1}\right)+1}-\delta_{1}\right)^{\alpha}}, \tau_{k\left(\delta_{1}\right)+1} \in\left[c_{1}, d_{1}\right]$, where $\tilde{r}=\max _{t \in\left[c_{1}, d_{1}\right] \cup\left[c_{2}, d_{2}\right]}\{r(t)\}$.

Proof For $t \in\left(\tau_{k}, \tau_{k+1}\right] \subset\left[c_{1}, d_{1}\right], k=k\left(c_{1}\right)+1, \ldots, k\left(d_{1}\right)-1$, there exists $\varsigma_{k} \in\left(\tau_{k}, t\right)$ such that

$$
x(t)-x\left(\tau_{k}^{+}\right)=x^{\prime}\left(\varsigma_{k}\right)\left(t-\tau_{k}\right) .
$$


In view of $x\left(\tau_{k}^{+}\right)>0$ and the monotone properties of $\Phi_{\alpha}(\cdot), r(t) \Phi_{\alpha}\left(x^{\prime}(t)\right)$ and $r(t)$, we obtain

$$
\Phi_{\alpha}(x(t))>\Phi_{\alpha}\left(x^{\prime}\left(\varsigma_{k}\right)\right) \Phi_{\alpha}\left(t-\tau_{k}\right) \geq \frac{r(t)}{r\left(\varsigma_{k}\right)} \Phi_{\alpha}\left(x^{\prime}(t)\right)\left(t-\tau_{k-1}\right)^{\alpha} .
$$

That is,

$$
\frac{r(t) \Phi_{\alpha}\left(x^{\prime}(t)\right)}{\Phi_{\alpha}(x(t))}<\frac{r\left(\varsigma_{k}\right)}{\left(t-\tau_{k}\right)^{\alpha}}
$$

Letting $t \rightarrow \tau_{k+1}^{-}$, we obtain conclusion (g). Using a similar analysis on $\left(c_{1}, \tau_{k\left(c_{1}\right)+1}\right]$ and $\left(\delta_{1}, \tau_{k\left(\delta_{1}\right)+1}\right]$, we can get $(\mathrm{h})$ and $(\mathrm{i})$. The proof is completed.

Lemma 2.4 Assume that, for any $T \geq t_{0}$, there exist $c_{2}, d_{2}, \delta_{2} \notin\left\{\tau_{k}\right\}$ such that $T<c_{2}-\sigma_{0}<$ $c_{2}<\delta_{2}<d_{2}$ and (6) hold. If $x(t)$ is a negative solution of $(1)$ and $u(t)$ is defined by

$$
u(t):=\frac{r(t) \Phi_{\alpha}\left(x^{\prime}(t)\right)}{\Phi_{\alpha}(x(t))}, \quad t \in\left[c_{2}, d_{2}\right]
$$

then the estimations (g)-(i) in Lemma 2.3 are correct with the replacement of $c_{1}, d_{1}$ and $\delta_{1}$ by $c_{2}, d_{2}$ and $\delta_{2}$, respectively.

The proof of Lemma 2.4 is similar to that of Lemma 2.3 and will be omitted.

Lemma 2.5 (cf. Lemma 1.1 in [22]) Let $\left\{\beta_{1}, \ldots, \beta_{n}\right\}$ be the $n$-tuple satisfying (A). Then there exists an $n$-tuple $\left(\eta_{1}, \eta_{2}, \ldots, \eta_{n}\right)$ satisfying
(i) $\sum_{i=1}^{n} \beta_{i} \eta_{i}=\alpha, \quad$ and
(ii) $\sum_{i=1}^{n} \eta_{i}=\lambda<1, \quad 0<\eta_{i}<1$.

In the following we will establish Kamenev type interval oscillation criteria for (1) by the idea of Philos [26]. For the research of Kamenev/Philos-type oscillation criteria for differential equations, see [27-31].

Let $E=\left\{(t, s): t_{0} \leq s \leq t\right\}, H_{1}, H_{2} \in C^{1}(E, \mathbb{R})$. Then a pair of functions $H_{1}, H_{2}$ is said to belong to a function set $\mathscr{H}$, denoted by $\left(H_{1}, H_{2}\right) \in \mathscr{H}$, if there exist $h_{1}, h_{2} \in L_{\text {loc }}(E, \mathbb{R})$ satisfying the following conditions:

( $\left.C_{1}\right) H_{1}(t, t)=H_{2}(t, t)=0, H_{1}(t, s)>0, H_{2}(t, s)>0$ for $t>s$;

$\left(C_{2}\right) \frac{\partial}{\partial t} H_{1}(t, s)=h_{1}(t, s) H_{1}(t, s), \frac{\partial}{\partial s} H_{2}(t, s)=h_{2}(t, s) H_{2}(t, s)$.

For convenience in the expression below, we also use the following notation:

$$
\int_{[c, d]}:=\int_{c}^{\tau_{k(c)+1}}+\sum_{k=k(c)+1}^{k(d)-1}\left(\int_{\tau_{k}}^{t_{k}}+\int_{t_{k}}^{\tau_{k+1}}\right)+\int_{\tau_{k(d)}}^{t_{k(d)}}+\int_{t_{k(d)}}^{d} .
$$


Lemma 2.6 Assume that the conditions of Lemma 2.1 hold. Let $x(t)$ be a positive solution of $(1)$ and $u(t)$ be defined by (7). Then, for any $\left(H_{1}, H_{2}\right) \in \mathscr{H}$, we have

$$
\begin{aligned}
& \int_{\left[c_{1}, \delta_{1}\right]} \psi(t) H_{1}\left(t, c_{1}\right) \frac{x^{\alpha}(t-\sigma(t))}{x^{\alpha}(t)} \mathrm{d} t \\
& \quad+\int_{c_{1}}^{\delta_{1}} H_{1}\left(t, c_{1}\right)\left[p_{0}(t)-\frac{r(t)}{(1+\alpha)^{1+\alpha}}\left|h_{1}\left(t, c_{1}\right)\right|^{1+\alpha}\right] \mathrm{d} t \\
& \leq \sum_{i=k\left(c_{1}\right)+1}^{k\left(\delta_{1}\right)} \frac{b_{i}^{\alpha}-a_{i}^{\alpha}}{a_{i}^{\alpha}} H_{1}\left(\tau_{i}, c_{1}\right) u\left(\tau_{i}\right)-H_{1}\left(\delta_{1}, c_{1}\right) u\left(\delta_{1}\right)
\end{aligned}
$$

and

$$
\begin{aligned}
& \int_{\left[\delta_{1}, d_{1}\right]} \psi(t) H_{2}\left(d_{1}, t\right) \frac{x^{\alpha}(t-\sigma(t))}{x^{\alpha}(t)} \mathrm{d} t \\
& \quad+\int_{\delta_{1}}^{d_{1}} H_{2}\left(d_{1}, t\right)\left[p_{0}(t)-\frac{r(t)}{(1+\alpha)^{1+\alpha}}\left|h_{2}\left(d_{1}, t\right)\right|^{1+\alpha}\right] \mathrm{d} t \\
& \leq \sum_{i=k\left(\delta_{1}\right)+1}^{k\left(d_{1}\right)} \frac{b_{i}^{\alpha}-a_{i}^{\alpha}}{a_{i}^{\alpha}} H_{2}\left(d_{1}, \tau_{i}\right) u\left(\tau_{i}\right)+H_{2}\left(d_{1}, \delta_{1}\right) u\left(\delta_{1}\right),
\end{aligned}
$$

where $\psi(t)=\eta_{0}^{-\eta_{0}}|f(t)|^{\eta_{0}} \prod_{i=1}^{n} \eta_{i}^{-\eta_{i}}\left(p_{i}(t)\right)^{\eta_{i}}$ with $\eta_{0}=1-\sum_{i=1}^{n} \eta_{i}$ and $\eta_{1}, \eta_{2}, \ldots, \eta_{n}$ are positive constants satisfying conditions of Lemma 2.5 .

Proof Differentiating $u(t)$ and in view of (1), we obtain, for $t \neq \tau_{k}$,

$$
\begin{aligned}
u^{\prime}(t)= & -\left[\sum_{i=1}^{n} p_{i}(t) \Phi_{\beta_{i}-\alpha}(x(t-\sigma(t)))+\frac{|f(t)|}{\Phi_{\alpha}(x(t-\sigma(t)))}\right] \frac{\Phi_{\alpha}(x(t-\sigma(t)))}{\Phi_{\alpha}(x(t))} \\
& -\frac{\alpha}{r^{1 / \alpha}(t)}|u(t)|^{1+1 / \alpha}-p_{0}(t) .
\end{aligned}
$$

Let

$$
v_{0}=\eta_{0}^{-1} \frac{|f(t)|}{\Phi_{\alpha}(x(t-\sigma(t)))}, \quad v_{i}=\eta_{i}^{-1} p_{i}(t) \Phi_{\beta_{i}-\alpha}(x(t-\sigma(t))), \quad i=1,2, \ldots, n,
$$

where $\eta_{1}, \eta_{2}, \ldots, \eta_{n}$ are chosen to satisfy conditions of Lemma 2.5 with $\eta_{0}=1-\sum_{i=1}^{n} \eta_{i}$ for given $\beta_{1}, \ldots, \beta_{n}$ and $\alpha$. Employing the arithmetic-geometric mean inequality (see [32])

$$
\sum_{i=0}^{n} \eta_{i} v_{i} \geq \prod_{i=0}^{n} v_{i}^{\eta_{i}}
$$

we have, from (12),

$$
u^{\prime}(t) \leq-\psi(t) \frac{x^{\alpha}(t-\sigma(t))}{x^{\alpha}(t)}-\frac{\alpha}{r^{1 / \alpha}(t)}|u(t)|^{1+1 / \alpha}-p_{0}(t)
$$

where

$$
\psi(t)=\eta_{0}^{-\eta_{0}}|f(t)|^{\eta_{0}} \prod_{i=1}^{n} \eta_{i}^{-\eta_{i}}\left(p_{i}(t)\right)^{\eta_{i}} .
$$


Multiplying both sides of (13) by $H_{1}\left(t, c_{1}\right)$ and integrating it from $c_{1}$ to $\delta_{1}$, we have

$$
\begin{aligned}
\int_{\left[c_{1}, \delta_{1}\right]} H_{1}\left(t, c_{1}\right) u^{\prime}(t) \mathrm{d} t \leq & -\int_{\left[c_{1}, \delta_{1}\right]} \psi(t) H_{1}\left(t, c_{1}\right) \frac{x^{\alpha}(t-\sigma(t))}{x^{\alpha}(t)} \mathrm{d} t \\
& -\alpha \int_{\left[c_{1}, \delta_{1}\right]} H_{1}\left(t, c_{1}\right) \frac{|u(t)|^{1+1 / \alpha}}{r^{1 / \alpha}(t)} \mathrm{d} t \\
& -\int_{c_{1}}^{\delta_{1}} H_{1}\left(t, c_{1}\right) p_{0}(t) \mathrm{d} t .
\end{aligned}
$$

Noticing that the impulse moments $\tau_{k\left(c_{1}\right)+1}, \tau_{k\left(c_{1}\right)+2}, \ldots, \tau_{k\left(\delta_{1}\right)}$ are in $\left[c_{1}, \delta_{1}\right]$ and using the integration by parts formula on the left-hand side of the above inequality, we obtain

$$
\begin{aligned}
\int_{\left[c_{1}, \delta_{1}\right]} H_{1}\left(t, c_{1}\right) u^{\prime}(t) \mathrm{d} t= & \sum_{i=k\left(c_{1}\right)+1}^{k\left(\delta_{1}\right)}\left(1-\frac{b_{i}^{\alpha}}{a_{i}^{\alpha}}\right) H_{1}\left(\tau_{i}, c_{1}\right) u\left(\tau_{i}\right)+H_{1}\left(\delta_{1}, c_{1}\right) u\left(\delta_{1}\right) \\
& -\int_{\left[c_{1}, \delta_{1}\right]} H_{1}\left(t, c_{1}\right) h_{1}\left(t, c_{1}\right) u(t) \mathrm{d} t .
\end{aligned}
$$

Substituting (15) into (14), we obtain

$$
\begin{aligned}
\int_{\left[c_{1}, \delta_{1}\right]} \psi(t) H_{1}\left(t, c_{1}\right) \frac{x^{\alpha}(t-\sigma(t))}{x^{\alpha}(t)} \mathrm{d} t \\
\leq \sum_{i=k\left(c_{1}\right)+1}^{k\left(\delta_{1}\right)}\left(\frac{b_{i}^{\alpha}}{a_{i}^{\alpha}}-1\right) H_{1}\left(\tau_{i}, c_{1}\right) u\left(\tau_{i}\right)-H_{1}\left(\delta_{1}, c_{1}\right) u\left(\delta_{1}\right) \\
\quad-\int_{c_{1}}^{\delta_{1}} p_{0}(t) H_{1}\left(t, c_{1}\right) \mathrm{d} t+\int_{\left[c_{1}, \delta_{1}\right]} H_{1}\left(t, c_{1}\right) V(u(t)) \mathrm{d} t
\end{aligned}
$$

where $V(u(t))=\left[\left|h_{1}\left(t, c_{1}\right)\right||u(t)|-\frac{\alpha}{r^{1 / \alpha}(t)}|u(t)|^{1+1 / \alpha}\right]$. We easily see that

$$
V(u(t)) \leq \sup _{u \in \mathbb{R}} V(u(t))=\frac{r(t)}{(1+\alpha)^{1+\alpha}}\left|h_{1}\left(t, c_{1}\right)\right|^{1+\alpha} .
$$

Thus, we obtain (10).

Multiplying both sides of (13) by $H_{2}\left(d_{1}, t\right)$ and using a similar analysis to the above, we can obtain (11). The proof is completed.

Lemma 2.7 Assume that the conditions of Lemma 2.2 hold. Let $x(t)$ be a negative solution of $(1)$ and $u(t)$ be defined by (8). Then for any $\left(H_{1}, H_{2}\right) \in \mathscr{H}$ we have

$$
\begin{aligned}
& \int_{\left[c_{2}, \delta_{2}\right]} \psi(t) H_{1}\left(t, c_{2}\right) \frac{x^{\alpha}(t-\sigma(t))}{x^{\alpha}(t)} \mathrm{d} t \\
& \quad+\int_{c_{2}}^{\delta_{2}} H_{1}\left(t, c_{2}\right)\left[p_{0}(t)-\frac{r(t)}{(1+\alpha)^{1+\alpha}}\left|h_{1}\left(t, c_{2}\right)\right|^{1+\alpha}\right] \mathrm{d} t \\
& \leq \sum_{i=k\left(c_{2}\right)+1}^{k\left(\delta_{2}\right)} \frac{b_{i}^{\alpha}-a_{i}^{\alpha}}{a_{i}^{\alpha}} H_{1}\left(\tau_{i}, c_{2}\right) u\left(\tau_{i}\right)-H_{1}\left(\delta_{2}, c_{2}\right) u\left(\delta_{2}\right)
\end{aligned}
$$


and

$$
\begin{aligned}
& \int_{\left[\delta_{2}, d_{2}\right]} \psi(t) H_{2}\left(d_{2}, t\right) \frac{x^{\alpha}(t-\sigma(t))}{x^{\alpha}(t)} \mathrm{d} t \\
& \quad+\int_{\delta_{2}}^{d_{2}} H_{2}\left(d_{2}, t\right)\left[p_{0}(t)-\frac{r(t)}{(1+\alpha)^{1+\alpha}}\left|h_{2}\left(d_{2}, t\right)\right|^{1+\alpha}\right] \mathrm{d} t \\
& \leq \sum_{i=k\left(\delta_{2}\right)+1}^{k\left(d_{2}\right)} \frac{b_{i}^{\alpha}-a_{i}^{\alpha}}{a_{i}^{\alpha}} H_{2}\left(d_{1}, \tau_{i}\right) u\left(\tau_{i}\right)-H_{2}\left(d_{2}, \delta_{2}\right) u\left(\delta_{2}\right),
\end{aligned}
$$

where $\psi(t)$ is defined as in Lemma 2.6.

The proof of Lemma 2.7 is similar to that of Lemma 2.6 and will be omitted.

For two constants $v_{1}, v_{2} \notin\left\{\tau_{k}\right\}$ with $v_{1}<\nu_{2}$ and $k\left(v_{1}\right)<k\left(v_{2}\right)$, using function $\varphi \in$ $C\left(\left[v_{1}, v_{2}\right], \mathbb{R}\right)$ and function $\phi \in C_{-}\left(\left[v_{1}, v_{2}\right], \mathbb{R}\right)$, we define a functional $Q: C\left(\left[v_{1}, v_{2}\right], \mathbb{R}\right) \rightarrow \mathbb{R}$ by

$$
Q_{\nu_{1}}^{\nu_{2}}[\varphi]:=\frac{\tilde{r}\left(b_{k\left(\nu_{1}\right)+1}^{\alpha}-a_{k\left(v_{1}\right)+1}^{\alpha}\right) \varphi\left(\tau_{k\left(\nu_{1}\right)+1}\right)}{a_{k\left(v_{1}\right)+1}^{\alpha}\left(\tau_{k\left(\nu_{1}\right)+1}-v_{1}\right)^{\alpha}}+\sum_{k=k\left(v_{1}\right)+2}^{k\left(v_{2}\right)} \frac{\tilde{r}\left(b_{k}^{\alpha}-a_{k}^{\alpha}\right) \varphi\left(\tau_{k}\right)}{a_{k}^{\alpha}\left(\tau_{k}-\tau_{k-1}\right)^{\alpha}}
$$

where $\sum_{m}^{n}=0$ if $m>n$, and a functional $L: C_{-}\left(\left[v_{1}, v_{2}\right], \mathbb{R}\right) \rightarrow \mathbb{R}$ by

$$
\begin{aligned}
& L_{v_{1}}^{\nu_{2}}[\phi]:=\int_{\nu_{1}}^{\tau_{k\left(\nu_{1}\right)+1}} \phi(t) \frac{\left(t-\tau_{k\left(\nu_{1}\right)}-\sigma(t)\right)^{\alpha}}{\left(t-\tau_{k\left(\nu_{1}\right)}\right)^{\alpha}} \mathrm{d} t \\
& +\sum_{k=k\left(v_{1}\right)+1}^{k\left(v_{2}\right)-1}\left[\int_{\tau_{k}}^{t_{k}} \phi(t) \frac{\left(t-\tau_{k}\right)^{\alpha}}{b_{k}^{\alpha}\left(t-\tau_{k}+\sigma(t)\right)^{\alpha}} \mathrm{d} t+\int_{t_{k}}^{\tau_{k+1}} \phi(t) \frac{\left(t-\tau_{k}-\sigma(t)\right)^{\alpha}}{\left(t-\tau_{k}\right)^{\alpha}} \mathrm{d} t\right] \\
& +\int_{\tau_{k\left(v_{2}\right)}}^{t_{k\left(v_{2}\right)}} \phi(t) \frac{\left(t-\tau_{k\left(v_{2}\right)}\right)^{\alpha}}{b_{k\left(v_{2}\right)}^{\alpha}\left(t-\tau_{k\left(v_{2}\right)}+\sigma(t)\right)^{\alpha}} \mathrm{d} t \\
& +\int_{t_{k\left(\nu_{2}\right)}}^{v_{2}} \phi(t) \frac{\left(t-\tau_{k\left(\nu_{2}\right)}-\sigma(t)\right)^{\alpha}}{\left(t-\tau_{k\left(\nu_{2}\right)}\right)^{\alpha}} \mathrm{d} t
\end{aligned}
$$

where $t_{k}$ are zero points of $D_{k}(t)$ on $\left[\tau_{k}, \tau_{k+1}\right]$ for $k=k\left(v_{1}\right)+1, \ldots, k\left(v_{2}\right)$.

For convenience in the expression below, we define, for $j=1,2$,

$$
\Pi_{c_{j}}^{\delta_{j}}\left[H_{1}\left(t, c_{j}\right)\right]:=L_{c_{j}}^{\delta_{j}}\left[\psi(t) H_{1}\left(t, c_{j}\right)\right]+\int_{c_{j}}^{\delta_{j}} H_{1}\left(t, c_{j}\right)\left[p_{0}(t)-\frac{r(t)}{(1+\alpha)^{1+\alpha}}\left|h_{1}\left(t, c_{j}\right)\right|^{1+\alpha}\right] \mathrm{d} t
$$

and

$$
\Pi_{\delta_{j}}^{d_{j}}\left[H_{2}\left(d_{j}, t\right)\right]:=L_{\delta_{j}}^{d_{j}}\left[\psi(t) H_{2}\left(d_{j}, t\right)\right]+\int_{\delta_{j}}^{d_{j}} H_{2}\left(d_{j}, t\right)\left[p_{0}(t)-\frac{r(t)}{(1+\alpha)^{1+\alpha}}\left|h_{2}\left(d_{j}, t\right)\right|^{1+\alpha}\right] \mathrm{d} t
$$

where $\psi(t)=\eta_{0}^{-\eta_{0}}|f(t)|^{\eta_{0}} \prod_{i=1}^{n} \eta_{i}^{-\eta_{i}}\left(p_{i}(t)\right)^{\eta_{i}}$. 
Theorem 2.1 Assume that, for any $T \geq t_{0}$, there exist $T<c_{1}-\sigma_{0}<c_{1}<d_{1} \leq c_{2}-\sigma_{0}<c_{2}<$ $d_{2}$ and (5) and (6) hold. If there exists a pair of $\left(H_{1}, H_{2}\right) \in \mathscr{H}$ such that

$$
\frac{\Pi_{c_{j}}^{\delta_{j}}\left[H_{1}\left(t, c_{j}\right)\right]}{H_{1}\left(\delta_{j}, c_{j}\right)}+\frac{\Pi_{\delta_{j}}^{d_{j}}\left[H_{2}\left(d_{j}, t\right)\right]}{H_{2}\left(d_{j}, \delta_{j}\right)}>\frac{Q_{c_{j}}^{\delta_{j}}\left[H_{1}\left(\cdot, c_{j}\right)\right]}{H_{1}\left(\delta_{j}, c_{j}\right)}+\frac{Q_{\delta_{j}}^{d_{j}}\left[H_{2}\left(d_{j}, \cdot\right)\right]}{H_{2}\left(d_{j}, \delta_{j}\right)}, \quad j=1,2,
$$

then (1) is oscillatory.

Proof Assume, to the contrary, that $x(t)$ is a nonoscillatory solution of (1). If $x(t)$ is a positive solution, we choose the interval $\left[c_{1}, d_{1}\right]$ to consider.

From Lemma 2.6, we obtain (10) and (11). Applying the estimation (a)-(f) into the left side, meanwhile (g) -(i) into the right side, of (10) and (11), we get

$$
\Pi_{c_{1}}^{\delta_{1}}\left[H_{1}\left(t, c_{1}\right)\right] \leq Q_{c_{1}}^{\delta_{1}}\left[H_{1}\left(\cdot, c_{1}\right)\right]-H_{1}\left(\delta_{1}, c_{1}\right) u\left(\delta_{1}\right)
$$

and

$$
\Pi_{\delta_{1}}^{d_{1}}\left[H_{2}\left(d_{1}, t\right)\right] \leq Q_{\delta_{1}}^{d_{1}}\left[H_{2}\left(d_{1}, \cdot\right)\right]+H_{2}\left(d_{1}, \delta_{1}\right) u\left(\delta_{1}\right)
$$

Dividing (21) and (22) by $H_{1}\left(\delta_{1}, c_{1}\right)$ and $H_{2}\left(d_{1}, \delta_{1}\right)$, respectively, and adding them, we get

$$
\frac{\Pi_{c_{1}}^{\delta_{1}}\left[H_{1}\left(t, c_{1}\right)\right]}{H_{1}\left(\delta_{1}, c_{1}\right)}+\frac{\Pi_{\delta_{1}}^{d_{1}}\left[H_{2}\left(d_{1}, t\right)\right]}{H_{2}\left(d_{1}, \delta_{1}\right)} \leq \frac{Q_{c_{1}}^{\delta_{1}}\left[H_{1}\left(\cdot, c_{1}\right)\right]}{H_{1}\left(\delta_{1}, c_{1}\right)}+\frac{Q_{\delta_{1}}^{d_{1}}\left[H_{2}\left(d_{1}, \cdot\right)\right]}{H_{2}\left(d_{1}, \delta_{1}\right)},
$$

which contradicts (20) for $j=1$.

If $x(t)$ is a negative solution of (1), we choose interval $\left[c_{2}, d_{2}\right]$ and can get a contradiction to (20) for $j=2$. The details will be omitted.

The proof is complete.

Remark 2.1 When $\sigma(t)=0$, i.e., the delay disappears and $\alpha=1$ in (1), Theorem 2.1 reduces to Theorem 2.2 in [1].

Remark 2.2 When $\sigma(t)=\sigma_{0}$, i.e., the delay is constant, Theorem 2.1 reduces to Theorem 2.8 in [2].

In Eq. (19), zero points $t_{k}$ of $D_{k}(t)$ appear at upper limit (or lower limit) of integrals. However, these zero points are generally not easy to solve from $D_{k}(t)=0$, which will lead to difficult in the calculation of (19). To overcome this difficulty we need to re-estimate $x(t-\sigma(t)) / x(t)$ on $\left(t_{k}, \tau_{k+1}\right],\left(\tau_{k}, t_{k}\right),\left(t_{k\left(d_{j}\right)}, d_{j}\right)$ and $\left(\tau_{k\left(d_{j}\right)}, t_{k\left(d_{j}\right)}\right)$ in Lemma 2.1 and Lemma 2.2.

If $x(t)$ is a positive solution of (1), from (a) in Lemma 2.1, we have, for $k=k\left(c_{1}\right)+$ $1, \ldots, k\left(d_{1}\right)-1, k \neq k\left(\delta_{1}\right)$,

$$
\frac{x(t-\sigma(t))}{x(t)}>\frac{t-\tau_{k}-\sigma(t)}{t-\tau_{k}}>\frac{t-\tau_{k}-\sigma(t)}{t}, \quad t \in\left(t_{k}, \tau_{k+1}\right] .
$$

From (b) in Lemma 2.1, we have, for $k=k\left(c_{1}\right)+1, \ldots, k\left(d_{1}\right)$,

$$
\frac{x(t-\sigma(t))}{x(t)}>\frac{t-\tau_{k}}{b_{k}\left(t+\sigma(t)-\tau_{k}\right)}>0>\frac{t-\tau_{k}-\sigma(t)}{t}, \quad t \in\left(\tau_{k}, t_{k}\right] .
$$


Combining (23) with (24), we obtain estimation of $x^{\alpha}(t-\sigma(t)) / x^{\alpha}(t)$ on $\left(\tau_{k}, \tau_{k+1}\right]$ for $k=$ $k\left(c_{1}\right)+1, \ldots, k\left(d_{1}\right)-1, k \neq k\left(\delta_{1}\right)$,

(a) $\frac{x^{\alpha}(t-\sigma(t))}{x^{\alpha}(t)}>\Phi_{\alpha}\left(\frac{t-\tau_{k}-\sigma(t)}{t}\right), \quad t \in\left(\tau_{k}, \tau_{k+1}\right]$.

Similarly, from (b) and (c) in Lemma 2.1, we have

(b) $\frac{x^{\alpha}(t-\sigma(t))}{x^{\alpha}(t)}>\Phi_{\alpha}\left(\frac{t-\tau_{k\left(\delta_{1}\right)}-\sigma(t)}{t}\right), \quad t \in\left(\tau_{k\left(\delta_{1}\right)}, t_{k\left(\delta_{1}\right)}\right] \cup\left(t_{k\left(\delta_{1}\right)}, \delta_{1}\right]$,

from (b) and (d) in Lemma 2.1, we have

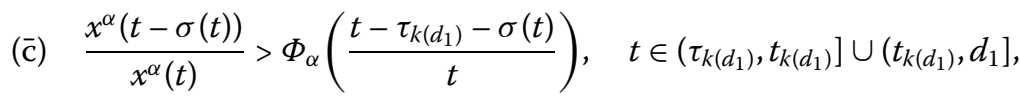

and from (e) and (f) in Lemma 2.1, we have

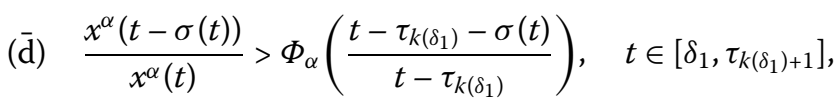

and

(可) $\frac{x^{\alpha}(t-\sigma(t))}{x^{\alpha}(t)}>\Phi_{\alpha}\left(\frac{t-\tau_{k\left(c_{1}\right)}-\sigma(t)}{t-\tau_{k\left(c_{1}\right)}}\right), \quad t \in\left[c_{1}, \tau_{k\left(c_{1}\right)+1}\right]$.

If $x(t)$ is a negative solution of (1), from Lemma 2.2, we can get similar estimations to the above for $t \in\left[c_{2}, d_{2}\right]$.

For convenience, we define functional $\widetilde{L}: C_{-}\left(\left[c_{j}, d_{j}\right], \mathbb{R}\right) \rightarrow \mathbb{R}$, for $j=1,2$, by

$$
\begin{aligned}
\widetilde{L}_{c_{j}}^{\delta_{j}}[\phi]:= & \int_{c_{j}}^{\tau_{k\left(c_{j}\right)+1}} \phi(t) \Phi_{\alpha}\left(\frac{t-\tau_{k\left(c_{j}\right)}-\sigma(t)}{t-\tau_{k\left(c_{j}\right)}}\right) \mathrm{d} t \\
& +\sum_{k=k\left(c_{j}\right)+1}^{k\left(\delta_{j}\right)-1} \int_{\tau_{k}}^{\tau_{k+1}} \phi(t) \Phi_{\alpha}\left(\frac{t-\tau_{k}-\sigma(t)}{t}\right) \mathrm{d} t \\
& +\int_{\tau_{k\left(\delta_{j}\right)}}^{\delta_{j}} \phi(t) \Phi_{\alpha}\left(\frac{t-\tau_{k\left(\delta_{j}\right)}-\sigma(t)}{t}\right) \mathrm{d} t
\end{aligned}
$$

and

$$
\begin{aligned}
\widetilde{L}_{\delta_{j}}^{d_{j}}[\phi]:= & \int_{\delta_{j}}^{\tau_{k\left(\delta_{j}\right)+1}} \phi(t) \Phi_{\alpha}\left(\frac{t-\tau_{k\left(\delta_{j}\right)}-\sigma(t)}{t-\tau_{k\left(\delta_{j}\right)}}\right) \mathrm{d} t \\
& +\sum_{k=k\left(\delta_{j}\right)+1}^{k\left(d_{j}\right)-1} \int_{\tau_{k}}^{\tau_{k+1}} \phi(t) \Phi_{\alpha}\left(\frac{t-\tau_{k}-\sigma(t)}{t}\right) \mathrm{d} t \\
& +\int_{\tau_{k\left(\delta_{j}\right)}}^{d_{j}} \phi(t) \Phi_{\alpha}\left(\frac{t-\tau_{k\left(\delta_{j}\right)}-\sigma(t)}{t}\right) \mathrm{d} t .
\end{aligned}
$$


Further, we define, for $j=1,2$,

$$
\widetilde{\Pi}_{c_{j}}^{\delta_{j}}\left[H_{1}\left(t, c_{j}\right)\right]:=\widetilde{L}_{c_{j}}^{\delta_{j}}\left[\psi(t) H_{1}\left(t, c_{j}\right)\right]+\int_{c_{j}}^{\delta_{j}} H_{1}\left(t, c_{j}\right)\left[p_{0}(t)-\frac{r(t)}{(1+\alpha)^{1+\alpha}}\left|h_{1}\left(t, c_{j}\right)\right|^{1+\alpha}\right] \mathrm{d} t
$$

and

$$
\widetilde{\Pi}_{c_{j}}^{\delta_{j}}\left[H_{2}\left(d_{j}, t\right)\right]:=\widetilde{L}_{c_{j}}^{\delta_{j}}\left[\psi(t) H_{2}\left(d_{j}, t\right)\right]+\int_{c_{j}}^{\delta_{j}} H_{2}\left(d_{j}, t\right)\left[p_{0}(t)-\frac{r(t)}{(1+\alpha)^{1+\alpha}}\left|h_{2}\left(d_{j}, t\right)\right|^{1+\alpha}\right] \mathrm{d} t,
$$

where $\psi(t)=\eta_{0}^{-\eta_{0}}|f(t)|^{\eta_{0}} \prod_{i=1}^{n} \eta_{i}^{-\eta_{i}}\left(p_{i}(t)\right)^{\eta_{i}}$.

Using similar proof method to that of Theorem 2.1 and applying estimations $(\overline{\mathrm{a}})-(\overline{\mathrm{e}})$, we can obtain following theorem.

Theorem 2.2 Assume that, for any $T \geq t_{0}$, there exist $T<c_{1}-\sigma_{0}<c_{1}<d_{1} \leq c_{2}-\sigma_{0}<c_{2}<$ $d_{2}$ and (5) and (6) hold. If there exists a pair of $\left(H_{1}, H_{2}\right) \in \mathscr{H}$ such that

$$
\frac{\widetilde{\Pi}_{c_{j}}^{\delta_{j}}\left[H_{1}\left(t, c_{j}\right)\right]}{H_{1}\left(\delta_{j}, c_{j}\right)}+\frac{\widetilde{\Pi}_{\delta_{j}}^{d_{j}}\left[H_{2}\left(d_{j}, t\right)\right]}{H_{2}\left(d_{j}, \delta_{j}\right)}>\frac{Q_{c_{j}}^{\delta_{j}}\left[H_{1}\left(\cdot, c_{j}\right)\right]}{H_{1}\left(\delta_{j}, c_{j}\right)}+\frac{Q_{\delta_{j}}^{d_{j}}\left[H_{2}\left(d_{j}, \cdot\right)\right]}{H_{2}\left(d_{j}, \delta_{j}\right)}, \quad j=1,2,
$$

then (1) is oscillatory.

\section{Example}

In this section, we give an example to illustrate the effectiveness and non-emptiness of our results.

Example 3.1 Consider the following equation:

$$
\begin{aligned}
& x^{\prime \prime}(t)+\mu_{1} p_{1}(t) \Phi_{\frac{5}{2}}(x(t-\sigma(t)))+\mu_{2} p_{2}(t) \Phi_{\frac{1}{2}}(x(t-\sigma(t)))=f(t), \quad t \neq \tau_{k}, \\
& x\left(\tau_{k}^{+}\right)=a_{k} x\left(\tau_{k}\right), \quad x^{\prime}\left(\tau_{k}^{+}\right)=b_{k} x^{\prime}\left(\tau_{k}\right), \quad k=1,2, \ldots
\end{aligned}
$$

where $\Phi_{*}(s)=|s|^{*-1} s, \sigma(t)=\frac{1}{3} \sin ^{2}(\pi t), \mu_{1}, \mu_{2}$ are positive constants and $\tau_{k}: \tau_{n, 1}=8 n+\frac{3}{2}$, $\tau_{n, 2}=8 n+\frac{5}{2}, \tau_{n, 3}=8 n+\frac{11}{2}, \tau_{n, 4}=8 n+\frac{13}{2}, n \in \mathbb{N}$.

Let

$$
p_{1}(t)=p_{2}(t)= \begin{cases}(t-8 n), & t \in[8 n, 8 n+3], \\ 3, & t \in[8 n+3,8 n+5], \\ (8 n+8-t), & t \in[8 n+5,8 n+8],\end{cases}
$$

and

$$
f(t)= \begin{cases}(t-8 n)(t-8 n-4)^{3}, & t \in[8 n, 8 n+4] \\ (t-8 n-4)^{3}(8 n+8-t), & t \in[8 n+4,8 n+8] .\end{cases}
$$

For any $t_{0}>0$, we choose $n$ large enough such that $t_{0}<8 n$ and let $\left[c_{1}, d_{1}\right]=[8 n+1,8 n+3]$, $\left[c_{2}, d_{2}\right]=[8 n+5,8 n+7], \delta_{1}=8 n+2$ and $\delta_{2}=8 n+6$. We see that there has a zero point of 
$D_{k}(t)$ on each interval of $\left[c_{1}, \delta_{1}\right],\left[\delta_{1}, d_{1}\right],\left[c_{2}, \delta_{2}\right]$ and $\left[\delta_{2}, d_{1}\right]$. By approximate calculation, we get $t_{1} \approx 8 n+1.709, t_{2} \approx 8 n+2.710, t_{3} \approx 8 n+5.709$ and $t_{4} \approx 8 n+6.710$. Moreover, from conditions $\alpha=1, \beta_{1}=5 / 2$ and $\beta_{2}=1 / 2$, we can choose $\eta_{1}=1 / 3, \eta_{1}=1 / 3$ and $\eta_{0}=$ $1-\eta_{1}-\eta_{2}=1 / 3$. So, the conditions of Lemma 2.5 are satisfied.

Letting $H_{1}(t, s)=H_{2}(t, s)=(t-s)^{2}$ and $h_{1}(t, s)=-h_{2}(t, s)=\frac{2}{t-s}$. By simple calculation, we have, for $t \in\left[c_{1}, \delta_{1}\right]$,

$$
\begin{aligned}
& \int_{c_{1}}^{\delta_{1}} H_{1}\left(t, c_{1}\right)\left[p_{0}(t)-\frac{r(t)}{(1+\alpha)^{1+\alpha}}\left|h_{1}\left(t, c_{1}\right)\right|^{1+\alpha}\right] \mathrm{d} t \\
& \quad=\int_{8 n+1}^{8 n+2}(t-8 n-1)^{2}\left(0-\frac{2^{2}}{2^{2}(t-8 n-1)^{2}}\right) \mathrm{d} t=-1 .
\end{aligned}
$$

Let

$$
\begin{aligned}
\phi_{1}(t) & :=\psi(t) H_{1}\left(t, c_{1}\right)=\eta_{0}^{-\eta_{0}}|f(t)|^{\eta_{0}} \prod_{i=1}^{2} \eta_{i}^{-\eta_{i}}\left(p_{i}(t)\right)^{\eta_{i}}\left(t-c_{1}\right)^{2} \\
& =3 \sqrt[3]{\mu_{1} \mu_{2}}(t-8 n)(t-8 n-4)(t-8 n-1)^{2} .
\end{aligned}
$$

Then

$$
\begin{aligned}
L_{c_{1}}^{\delta_{1}}\left[\phi_{1}(t)\right]= & \int_{8 n+1}^{8 n+\frac{3}{2}} \phi_{1}(t) \frac{t-8 n+\frac{3}{2}-\frac{1}{3} \sin ^{2}(\pi t)}{t-8 n+\frac{3}{2}} \mathrm{~d} t \\
& +\int_{8 n+\frac{3}{2}}^{t_{1}} \phi_{1}(t) \frac{t-8 n-\frac{3}{2}}{b_{n, 1}\left(t-8 n-\frac{3}{2}+\frac{1}{3} \sin ^{2}(\pi t)\right)} \mathrm{d} t \\
& +\int_{t_{1}}^{8 n+2} \phi_{1}(t) \frac{t-8 n-\frac{3}{2}-\frac{1}{3} \sin ^{2}(\pi t)}{t-8 n-\frac{3}{2}} \mathrm{~d} t \\
= & 3 \sqrt[3]{\mu_{1} \mu_{2}}\left(\int_{1}^{\frac{3}{2}} \frac{t(4-t)(t-1)^{2}\left(t+\frac{3}{2}-\frac{1}{3} \sin ^{2}(\pi t)\right)}{t+\frac{3}{2}} \mathrm{~d} t\right. \\
& +\int_{\frac{3}{2}}^{1.709} \frac{t(4-t)(t-1)^{2}\left(t-\frac{3}{2}\right)}{b_{n, 1}\left(t-\frac{3}{2}+\frac{1}{3} \sin ^{2}(\pi t)\right)} \mathrm{d} t \\
& \left.+\int_{1.709}^{2} \frac{t(4-t)(t-1)^{2}\left(t-\frac{3}{2}-\frac{1}{3} \sin ^{2}(\pi t)\right)}{t-\frac{3}{2}} \mathrm{~d} t\right) \\
\approx & 3 \sqrt[3]{\mu_{1} \mu_{2}}\left(2.373+\frac{0.2551}{b_{n, 1}}\right) .
\end{aligned}
$$

Therefore,

$$
\Pi_{c_{1}}^{\delta_{1}}\left[H_{1}\left(t, c_{1}\right)\right]=3 \sqrt[3]{\mu_{1} \mu_{2}}\left(2.373+\frac{0.2551}{b_{n, 1}}\right)-1 .
$$

Similarly, for $t \in\left[\delta_{1}, d_{1}\right]$, we have

$$
\begin{aligned}
& \phi_{2}(t):=\psi(t) H_{2}\left(d_{1}, t\right)=3 \sqrt[3]{\mu_{1} \mu_{2}}(t-8 n)(8 n+4-t)(8 n+3-t)^{2}, \\
& \int_{\delta_{1}}^{d_{1}} H_{2}\left(d_{1}, t\right)\left[p_{0}(t)-\frac{r(t)}{(1+\alpha)^{1+\alpha}}\left|h_{2}\left(d_{1}, t\right)\right|^{1+\alpha}\right] \mathrm{d} t=-1,
\end{aligned}
$$


and

$$
\begin{aligned}
L_{\delta_{1}}^{d_{1}}\left[\phi_{2}(t)\right]= & 3 \sqrt[3]{\mu_{1} \mu_{2}}\left(\int_{2}^{\frac{5}{2}} \frac{t(4-t)(3-t)^{2}\left(t-\frac{3}{2}-\frac{1}{3} \sin ^{2}(\pi t)\right)}{t-\frac{3}{2}} \mathrm{~d} t\right. \\
& +\int_{\frac{5}{2}}^{2.71} \frac{t(4-t)(3-t)^{2}\left(t-\frac{5}{2}\right)}{b_{n, 2}\left(t-\frac{5}{2}+\frac{1}{3} \sin ^{2}(\pi t)\right)} \mathrm{d} t \\
& \left.+\int_{2.71}^{3} \frac{t(4-t)(3-t)^{2}\left(t-\frac{5}{2}-\frac{1}{3} \sin ^{2}(\pi t)\right)}{t-\frac{5}{2}} \mathrm{~d} t\right) \\
\approx & 3 \sqrt[3]{\mu_{1} \mu_{2}}\left(2.964+\frac{0.078}{b_{n, 2}}\right) .
\end{aligned}
$$

Therefore,

$$
\Pi_{\delta_{1}}^{d_{1}}\left[H_{2}\left(d_{1}, t\right)\right]=3 \sqrt[3]{\mu_{1} \mu_{2}}\left(2.964+\frac{0.078}{b_{n, 2}}\right)-1
$$

Since

$$
H_{1}\left(\delta_{1}, c_{1}\right)=\left(\delta_{1}-c_{1}\right)^{2}=1, \quad H_{2}\left(d_{1}, \delta_{1}\right)=\left(d_{1}-\delta_{1}\right)^{2}=1,
$$

the left-hand side of inequality (20) is

$$
\frac{\Pi_{c_{1}}^{\delta_{1}}\left[H_{1}\left(t, c_{1}\right)\right]}{H_{1}\left(\delta_{1}, c_{1}\right)}+\frac{\Pi_{\delta_{1}}^{d_{1}}\left[H_{2}\left(d_{1}, t\right)\right]}{H_{2}\left(d_{1}, \delta_{1}\right)} \approx 3 \sqrt[3]{\mu_{1} \mu_{2}}\left(5.337+\frac{0.255}{b_{n, 1}}+\frac{0.078}{b_{n, 2}}\right)-2 .
$$

Because $\tilde{r}_{1}=\tilde{r}_{2}=1, \tau_{k\left(c_{1}\right)+1}=\tau_{k\left(\delta_{1}\right)}=\tau_{n, 1}=8 n+\frac{3}{2} \in\left(c_{1}, \delta_{1}\right)$ and $\tau_{k\left(\delta_{1}\right)+1}=\tau_{k\left(d_{1}\right)}=\tau_{n, 2}=$ $8 n+\frac{5}{2} \in\left(\delta_{1}, d_{1}\right)$, it is easy to see that the right-hand side of inequality (20) for $j=1$ is

$$
\frac{Q_{c_{1}}^{\delta_{1}}\left[H_{1}\left(\cdot, c_{1}\right)\right]}{H_{1}\left(\delta_{1}, c_{1}\right)}+\frac{Q_{\delta_{1}}^{d_{1}}\left[H_{2}\left(d_{1}, \cdot\right)\right]}{H_{2}\left(d_{1}, \delta_{1}\right)}=\frac{b_{n, 1}-a_{n, 1}}{4 a_{n, 1}}+\frac{b_{n, 2}-a_{n, 2}}{4 a_{n, 2}} .
$$

Thus (20) is satisfied with $j=1$ if

$$
3 \sqrt[3]{\mu_{1} \mu_{2}}\left(5.337+\frac{0.255}{b_{n, 1}}+\frac{0.078}{b_{n, 2}}\right)>2+\frac{b_{n, 1}-a_{n, 1}}{4 a_{n, 1}}+\frac{b_{n, 2}-a_{n, 2}}{4 a_{n, 2}} .
$$

When $j=2$, with the same argument as above we see that the left-hand side of inequality (20) is

$$
\begin{aligned}
& \frac{\Pi_{c_{2}}^{\delta_{2}}\left[H_{1}\left(t, c_{2}\right)\right]}{H_{1}\left(\delta_{2}, c_{2}\right)}+\frac{\Pi_{\delta_{2}}^{d_{2}}\left[H_{2}\left(d_{2}, t\right)\right]}{H_{2}\left(d_{2}, \delta_{2}\right)} \\
& =3 \sqrt[3]{\mu_{1} \mu_{2}}\left(\int_{1}^{\frac{3}{2}} \frac{t(4-t)(t-1)^{2}\left(t+\frac{3}{2}-\frac{1}{3} \sin ^{2}(\pi t)\right)}{t+\frac{3}{2}} \mathrm{~d} t\right. \\
& \quad+\int_{\frac{3}{2}}^{1.709} \frac{t(4-t)(t-1)^{2}\left(t-\frac{3}{2}\right)}{b_{n, 3}\left(t-\frac{3}{2}+\frac{1}{3} \sin ^{2}(\pi t)\right)} \mathrm{d} t \\
& \quad+\int_{1.709}^{2} \frac{t(4-t)(t-1)^{2}\left(t-\frac{3}{2}-\frac{1}{3} \sin ^{2}(\pi t)\right)}{t-\frac{3}{2}} \mathrm{~d} t
\end{aligned}
$$




$$
\begin{aligned}
& +\int_{2}^{\frac{5}{2}} \frac{t(4-t)(3-t)^{2}\left(t-\frac{3}{2}-\frac{1}{3} \sin ^{2}(\pi t)\right)}{t-\frac{3}{2}} \mathrm{~d} t \\
& +\int_{\frac{5}{2}}^{2.71} \frac{t(4-t)(3-t)^{2}\left(t-\frac{5}{2}\right)}{b_{n, 4}\left(t-\frac{5}{2}+\frac{1}{3} \sin ^{2}(\pi t)\right)} \mathrm{d} t \\
& \left.+\int_{2.71}^{3} \frac{t(4-t)(3-t)^{2}\left(t-\frac{5}{2}-\frac{1}{3} \sin ^{2}(\pi t)\right)}{t-\frac{5}{2}} \mathrm{~d} t\right)-2 \\
& \approx 3 \sqrt[3]{\mu_{1} \mu_{2}}\left(5.337+\frac{0.255}{b_{n, 3}}+\frac{0.078}{b_{n, 4}}\right)-2,
\end{aligned}
$$

and the right-hand side of inequality (20) is

$$
\frac{Q_{c_{2}}^{\delta_{2}}\left[H_{1}\left(\cdot, c_{2}\right)\right]}{H_{2}\left(\delta_{2}, c_{2}\right)}+\frac{Q_{\delta_{2}}^{d_{2}}\left[H_{2}\left(d_{2}, \cdot\right)\right]}{H_{2}\left(d_{2}, \delta_{2}\right)}=\frac{b_{n, 3}-a_{n, 3}}{4 a_{n, 3}}+\frac{b_{n, 4}-a_{n, 4}}{4 a_{n, 4}} .
$$

Therefore, (20) is satisfied for $j=2$, if

$$
3 \sqrt[3]{\mu_{1} \mu_{2}}\left(5.337+\frac{0.255}{b_{n, 1}}+\frac{0.078}{b_{n, 2}}\right)>2+\frac{b_{n, 3}-a_{n, 3}}{4 a_{n, 3}}+\frac{b_{n, 4}-a_{n, 4}}{4 a_{n, 4}} .
$$

Hence, by Theorem 2.1, Eq. (26) is oscillatory, if

$$
\left\{\begin{array}{l}
3 \sqrt[3]{\mu_{1} \mu_{2}}\left(5.337+\frac{0.255}{b_{n, 1}}+\frac{0.078}{b_{n, 2}}\right)>2+\frac{b_{n, 1}-a_{n, 1}}{4 a_{n, 1}}+\frac{b_{n, 2}-a_{n, 2}}{4 a_{n, 2}}, \\
3 \sqrt[3]{\mu_{1} \mu_{2}}\left(5.337+\frac{0.255}{b_{n, 1}}+\frac{0.078}{b_{n, 2}}\right)>2+\frac{b_{n, 3}-a_{n, 3}}{4 a_{n, 3}}+\frac{b_{n, 4}-a_{n, 4}}{4 a_{n, 4}}
\end{array}\right.
$$

\section{Acknowledgements}

The authors sincerely thank the editors and reviewers for their valuable suggestions and useful comments that have led to the present improved version of the original manuscript.

\section{Funding}

This work has been supported by the NNSF of China [Grant No. 11561019], the Science and Technology Planning Projects of Guangdong Province [Grant No. 2017A030303085] and of Zhanjiang City [Grant No. 2016A01001], the Key Subject Program of Lingnan Normal University [Grant No. 1171518004] and the Special Funds for the Cultivation of Guangdong College Students' Scientific and Technological Innovation [Grant No. pdjh0304].

\section{Availability of data and materials}

Not applicable.

\section{Competing interests}

The authors declare that they have no competing interests.

\section{Authors' contributions}

Each of the authors, XZ, CL and RC contributed to each part of this study equally and read and approved the final version of the manuscript.

\section{Author details}

'Department of Mathematics, Lingnan Normal University, Zhanjiang, P.R. China. ${ }^{2}$ Department of Mathematics, Guangdong Ocean University, Zhanjiang, P.R. China.

\section{Publisher's Note}

Springer Nature remains neutral with regard to jurisdictional claims in published maps and institutional affiliations.

Received: 26 July 2018 Accepted: 12 December 2018 Published online: 18 January 2019

\section{References}

1. Liu, X., Xu, Z:: Oscillation criteria for a forced mixed type Emden-Fowler equation with impulses. Appl. Math. Comput. 215, 283-291 (2009) 
2. Guo, Z., Zhou, X., Wang, W.S.: Interval oscillation criteria of second order mixed nonlinear impulsive differential equations with delay. Abstr. Appl. Anal. 2012, Article ID 351709 (2012)

3. Wong, J.S.W.: Oscillation criteria for a forced second order linear differential equation. J. Math. Anal. Appl. 231, 235-240 (1999)

4. Li, W.T.: Interval oscillation theorems for second-order quasi-linear nonhomogeneous differential equations with damping. Appl. Math. Comput. 147, 753-763 (2004)

5. Wong, Q.: Interval criteria for oscillation of second-order nonlinear differential equations. J. Comput. Appl. Math. 205, 231-238 (2007)

6. Wong, Q., Yang, Q.: Interval criteria for oscillation of second-order half-linear differential equations. J. Math. Anal. Appl. 291, 224-236 (2004)

7. Çakmak, D.: Interval integral averaging technique for the interval oscillation criteria of certain second-order nonlinear differential equations. J. Math. Anal. Appl. 300, 408-425 (2004)

8. Liu, X., Xu, Z: Oscillation of a forced super-linear second order differential equation with impulses. Comput. Math. Appl. 53, 1740-1749 (2007)

9. Özbekler, A., Zafer, A.: Forced oscillation of super-half-linear impulsive differential equations. Comput. Math. Appl. 54, 785-792 (2007)

10. Özbekler, A.z Zafer, A.: Interval criteria for the forced oscillation of super-half-linear differential equations under impulse effects. Math. Comput. Model. 50, 59-65 (2009)

11. Zhou, X., Guo, Z., Wang, W.S.: Interval oscillation criteria for nonlinear impulsive delay differential equations with damping term. Appl. Math. Comput. 249, 32-44 (2014)

12. Zhou, X., Wang, W.S.: Interval oscillation criteria for nonlinear impulsive differential equations with variable delay. Electron. J. Qual. Theory Differ. Equ. 2016, 101 (2016)

13. Agarwal, R.P., Anderson, D.R., Zafer, A.: Interval oscillation criteria for second-order forced delay dynamic equations with mixed nonlinearities. Comput. Math. Appl. 59, 977-993 (2010)

14. Sun, Y.G., Wong, J.S.W.: Oscillation criteria for second order forced ordinary differential equations with mixed nonlinearities. J. Math. Anal. Appl. 334, 549-560 (2007)

15. Sun, Y.G., Meng, F.W.: Interval criteria for oscillation of second-order differential equations with mixed nonlinearities. Appl. Math. Comput. 15, 375-381 (2008)

16. Nazr, A.H.: Sufficient conditions for the oscillation of forced super-linear second-order differential equations with oscillatory potential. Proc. Am. Math. Soc. 126, 123-125 (1998)

17. Kong, Q.: Interval criteria for oscillation of second-order linear ordinary differential equations. J. Math. Anal. Appl. 229, 258-270 (1999)

18. Li, T., Rogovchenko, Y.V.: Oscillation of second-order neutral differential equations. Math. Nachr. 288, 1150-1162 (2015)

19. Li, T., Rogovchenko, Y.V.: Oscillation criteria for second-order superlinear Emden-Fowler neutral differential equations. Monatshefte Math. 184, 489-500 (2017)

20. Li, T., Rogovchenko, Y.V.: On asymptotic behavior of solutions to higher-order sublinear Emden-Fowler delay differential equations. Appl. Math. Lett. 67, 53-59 (2017)

21. Yang, Q.: Interval oscillation criteria for a forced second order nonlinear ordinary differential equations with oscillatory potential. Appl. Math. Comput. 135, 49-64 (2003)

22. Özbekler, A.: Oscillation of solutions of second order mixed nonlinear differential equations under impulsive perturbations. Comput. Math. Appl. 61, 933-940 (2011)

23. Huang, M., Feng, W.: Forced oscillations for second order delay differential equations with impulses. Comput. Math. Appl. 59, 18-30 (2010)

24. Guo, Z., Zhou, X., Ge, W.: Interval oscillation criteria for second-order forced impulsive differential equations with mixed nonlinearities. J. Math. Anal. Appl. 381, 187-201 (2011)

25. Zhou, X., Wang, W.S., Chen, R.Y.: Interval oscillation criteria for nonlinear impulsive differential equations with variable delay. Appl. Math. Lett. 75, 89-95 (2018)

26. Philos, C.G.: Oscillation theorems for linear differential equations of second order. Arch. Math. 53, 482-492 (1989)

27. Agarwal, R.P., Zhang, C., Li, T.: New Kamenev-type oscillation criteria for second-order nonlinear advanced dynamic equations. Appl. Math. Comput. 225, 822-828 (2013)

28. Agarwal, R.P., Bohner, M., Li, T., Zhang, C.: A Philos-type theorem for third-order nonlinear retarded dynamic equations. Appl. Math. Comput. 249, 527-531 (2014)

29. Bohner, M., Li, T.: Kamenev-type criteria for nonlinear damped dynamic equations. Sci. China Math. 58, 1445-1452 (2015)

30. Bohner, M., Hassan, T.S., Li, T.: Fite-Hille-Wintner-type oscillation criteria for second-order half-linear dynamic equations with deviating arguments. Indag. Math. 29, 548-560 (2018)

31. Yang, J., Li, T.: A Philos-type theorem for second-order neutral delay dynamic equations with damping. Adv. Differ. Equ. 2016, 44 (2016)

32. Hardy, G.H., Littlewood, J.E., Polya, G.: Inequalities. Cambridge University Press, Cambridge (1964) 